\title{
A Vehicle Fuel Consumption Model on Reconstructed Roads Based on the Roughness and Its Measurement Method
}

\author{
Qiang Liu $\mathbb{D}^{1,2}$ Jianguang Xie, ${ }^{1}$ and Zhixiang Zhang ${ }^{2}$ \\ ${ }^{1}$ Nanjing University of Aeronautics and Astronautics, Nanjing 211106, Jiangsu Province, China \\ ${ }^{2}$ Jiangsu Sinoroad Engineering Research Institute Co., Ltd., Nanjing 211008, Jiangsu Province, China \\ Correspondence should be addressed to Qiang Liu; 1154228184@qq.com
}

Received 2 September 2020; Revised 10 May 2021; Accepted 29 June 2021; Published 21 July 2021

Academic Editor: Fan Gu

Copyright (c) 2021 Qiang Liu et al. This is an open access article distributed under the Creative Commons Attribution License, which permits unrestricted use, distribution, and reproduction in any medium, provided the original work is properly cited.

The fuel consumption model for a vehicle forms a basis and method for evaluating the social benefit of road reconstruction. Based on the theoretical fuel consumption model of vehicles, the influence mechanism of the apparent parameters of the road surface on the fuel consumption of vehicles and their sensitivity was analysed. The baseline state was defined on the basis of the roughness, a parameter with significant influence. In addition, a method for acquiring fuel consumption parameters was proposed to establish a one-parameter relational model for the roughness and fuel consumption of vehicles in the baseline state based on measured data. Moreover, by considering the effects of the friction coefficient, deflection of the road surface, and vehicle speed on the fuel consumption, a modified model for the fuel consumption of vehicles applicable to road reconstruction was established. Finally, a method for measuring the energy-saving benefit of vehicles was proposed based on the characteristics of reconstructed roads. The research provides a basis for evaluating the social benefit acquired from reconstructed roads.

\section{Introduction}

Fuel consumption for vehicles is an important part of energy consumption in the life of a highway. The reduction of the fuel consumption saves energy and reduces emissions within the life cycle of a road $[1,2]$. According to the existing literature, fuel consumption is mainly analysed from the perspective of feasibility of road engineering, mainly evaluating the improvement of road networks and paths for user travel based on road projects. With the constant improvement of people's demand for comfortable and convenient travel, reconstruction or major repair of roads for improving traffic capacity and level of service has gradually become the focus of road management departments. Most previous research studies are based on the evaluation of user benefits from newly built highways, while little has been done on the evaluation of users' social benefits arising from reconstructed roads.

How to evaluate the influence of the reconstructed roads on the fuel consumption of road users' vehicles is an important approach when exploring the social benefit from road reconstruction activities. To calculate users' energysaving benefit, a fuel consumption model was established based on the measured data of the fuel consumption of vehicles by investigating the influence of the roughness of the road surface on the fuel consumption of vehicles. Furthermore, a calculation method for the user benefit in fuel consumption from reconstructed roads was proposed, aiming to provide a reference for evaluating users' benefit in fuel consumption and their social benefit from reconstructed roads.

\section{The Influence Mechanism of Road Roughness on the Fuel Consumption of Vehicles}

According to existing literature, the road roughness exerts the most significant influence on the fuel consumption of vehicles among the characteristic parameters of the road surface [3-5]. Due to the difference of wavelengths of road surface structures, the road surface can be partitioned into different types. The section with the wavelength larger than 
$0.5 \mathrm{~m}$ can be characterised by using roughness. The influence of the roughness on the wheel driving can generally be characterised as the effect of different wave amplitudes of road surfaces. Generally, the road surface with a larger international roughness index (IRI) has a larger wave amplitude [6-9]. The road surface-wheel mechanical model can be simplified as shown in Figure 1.

Based on the above simplified mechanical model, the method for calculating the roughness and couple of rolling resistance is proposed, as shown in the following formula:

$$
M_{f}=F_{R} \cdot \sin \alpha \cdot d \cdot \delta=F_{T} \cdot \tan \alpha \cdot d \cdot \delta,
$$

where $M_{f}, F_{T}, F_{R}, \alpha, \delta$, and $d$ refer to the couple of rolling resistance, traction force on rolling wheels, normal force on the rolling surface, included angle between the rolling section and road surface, distance of the component force in the direction of action of the self-weight component of the normal force $F_{R}$ to the origin, and wave amplitude of roughness, respectively.

Given fixed $\delta$, the higher the roughness of the road surface, the larger the wave amplitude $d$, correspondingly, the larger the value of $\alpha$, and the larger the couple of rolling resistance $\mathrm{M}_{f}$ with fixed $F_{T}$, that is, the greater the required work of the vehicle brake to overcome the couple of rolling resistance $\mathrm{M}_{f}$ and the larger the fuel consumption. In terms of $\delta$, it is difficult to measure the roughness of the road surface by using quantitative indices; however, theoretically, the larger the wave amplitude $d$, the larger the arm of force $\delta$. This indicates that a road surface with a higher roughness exerts a greater influence on the fuel consumption.

\section{The Relationship between the Roughness and Fuel Consumption in the Baseline State}

The reference state of the model was defined based on the measured data, and the relationship between the roughness and fuel consumption of vehicles in the baseline state was proposed. Furthermore, the model was modified by incorporating other parameters.

3.1. Acquisition of Fuel Consumption Parameters. To improve the accuracy of the model, the data on fuel consumption of vehicles and characteristic parameters of the road surfaces were measured $[10,11]$. The fuel consumption of vehicles was tested by utilising a GPS engine fuel consumption detection system. By combining the single-way measurement method of fuel inlet monitoring, the fuel consumption of the vehicle engine was collected in real time; moreover, the data on fuel consumption and location information were sent through a signal acquisition card and GPS, and they were processed and displayed after being sent to the server through a wireless transmission module. The measurement accuracy reached 3\%o, as shown in Figure 2. The characteristic parameters of the road surfaces were collected by using a standard test method recommended in related standards $[12,13]$.

During the test, after setting the corresponding driving speed on the tested target section, the mileages of the preset

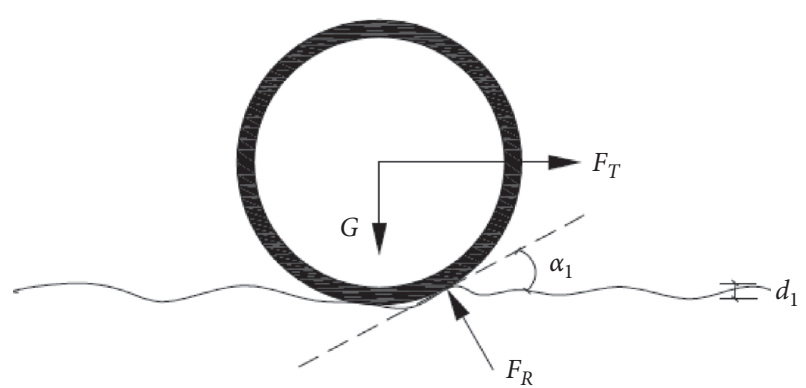

FIgURE 1: Road surface-wheel mechanical model.

positions and corresponding arrival times were recorded. It should be guaranteed that the speed varied within 3\% in the driving process; in addition, it was necessary to reduce the influence of lane changing and overtaking as far as possible and avoid testing in rainy and windy weather.

3.2. Sample Sections for the Test. Six sample sections with the total length of $32 \mathrm{~km}$ were selected nationwide (Table 1).

\subsection{Fuel Consumption Model in the Baseline State}

3.3.1. Definition of the Baseline State. The parameter changes triggered by activity characteristics of the reconstructed roads are mainly related to changes in parameters (including roughness, deflection, and friction coefficient) of the road surface layer, which are irrelevant to the external climate environment. Therefore, to explore the relationship between roughness and fuel consumption, it was necessary to define the external factors to avoid the influence of the change of the external factors on the test analysis. Moreover, to decrease the additional testing workload, the changing parameters (such as deflection and friction coefficient) were defined within high-probability value ranges based on samples, expecting to reduce the influence of the other parameters on the model. Therefore, it was necessary to acquire and analyse the data by selecting a proper working condition, which was defined as the baseline state of the road surface. By analysing the characteristic data of the road surfaces within the sample sections, the baseline state was defined (Table 2).

3.3.2. Calibration of Vehicle Type and Vehicle Speed. The fuel consumptions of different types of vehicles vary: nevertheless, considering the connection and comparison with relevant research achievements, vehicles were divided into five types (i.e., minibus, coach, light-duty truck, mediumduty truck, and heavy-duty truck) according to the need.

As shown in Figure 3, the average driving speeds of different types of vehicles within the sample sections were determined by installing a vehicle pretest system in the test sections, which were also the basis for model analysis [14]. The section data collected by the installed vehicle pretest device were summarised and analysed. Moreover, the driving speeds, vehicle types, and axle types were recorded by using sensors to calculate the average driving speeds of 


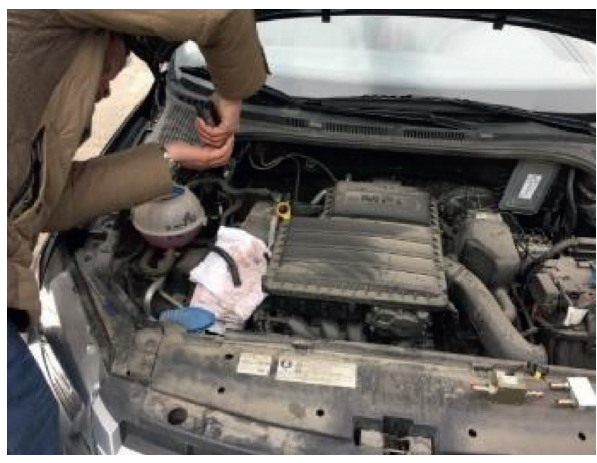

(a)

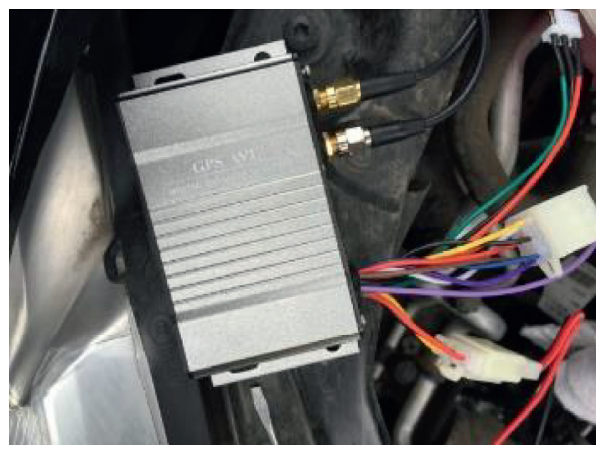

(c)

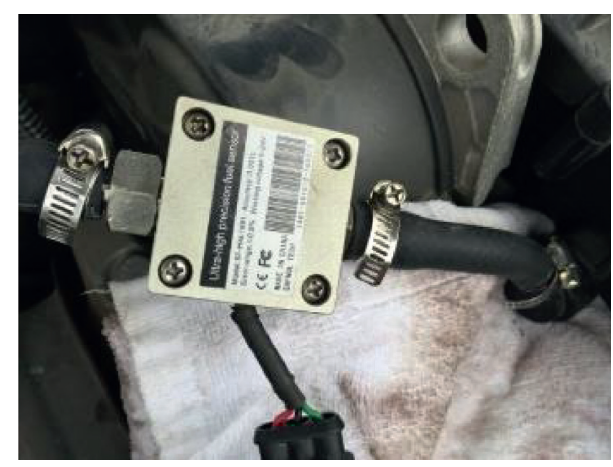

(b)

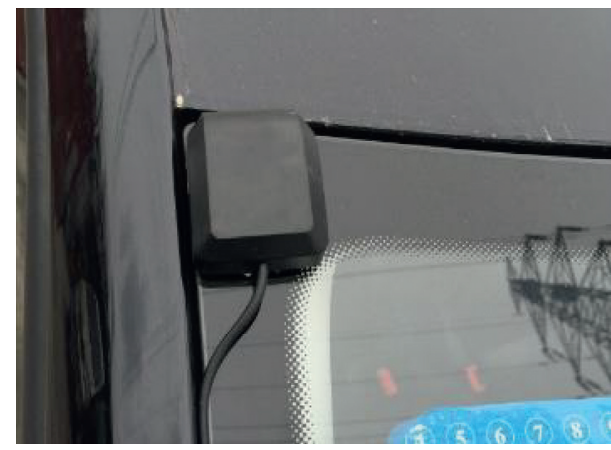

(d)

FIGURE 2: Collection scheme of fuel consumption parameters. (a) Oil-pipe reconstruction. (b) Fuel consumption sensor. (c) Data acquisition device. (d) GPS positioning.

TABle 1: Survey of sample sections.

\begin{tabular}{lccccc}
\hline Serial number & Region & Road class & Number of lanes & Length of the sample section $(\mathrm{km})$ & Number of samples \\
\hline 1 & Jiangsu province & Highway & Six two-way lanes & 5 & 3 \\
2 & Jiangsu province & Highway & Four two-way lanes & 5 & 7 \\
3 & Hebei province & Highway & Four two-way lanes & 10 & 3 \\
4 & Jiangsu province & Highway & Four two-way lanes & 5 & 4 \\
5 & Jiangsu province & First-class highway & Four two-way lanes & 4 & 3 \\
6 & Beijing & Highway & Four two-way lanes & & 3 \\
\hline
\end{tabular}

TABLE 2: Definition of the baseline state.

\begin{tabular}{lccc}
\hline Serial number & Item & Definition & Remark \\
\hline 1 & Climate environment & Rainless weather with wind speed lower than $5 \mathrm{~m} / \mathrm{s}$ & Plain lightly undulate area \\
2 & Longitudinal slope & Average longitudinal slope less than $2 \%$ & Nonrestraint \\
3 & Roughness & - & \\
4 & Deflection & $8 \sim 10(0.01 \mathrm{~mm})$ & \\
5 & SFC & $50 \sim 52$ & \\
\hline
\end{tabular}

different types of vehicles within controlled sections. According to the speed limit, the average speed selections of different types of vehicles are listed in Table 3: these were taken as the calibrated speeds for fuel consumption test purposes.

3.3.3. Sample Data on the Fuel Consumption Model. The fuel consumptions of different types of vehicles within the above sample sections were tested based on the calibrated speeds. The cumulative fuel consumptions of vehicles within the unit section were recorded; moreover, the average fuel consumptions were calculated according to the mileage. The data pertaining to the average fuel consumptions within the sample sections are listed in Table 4.

3.3.4. Establishment of the Relationship between Roughness and Fuel Consumption. The fuel consumptions of different types of vehicles and road roughness were fitted by using the linear function, with all correlation coefficients exceeding 0.8. In the fitting process, some models showed better 


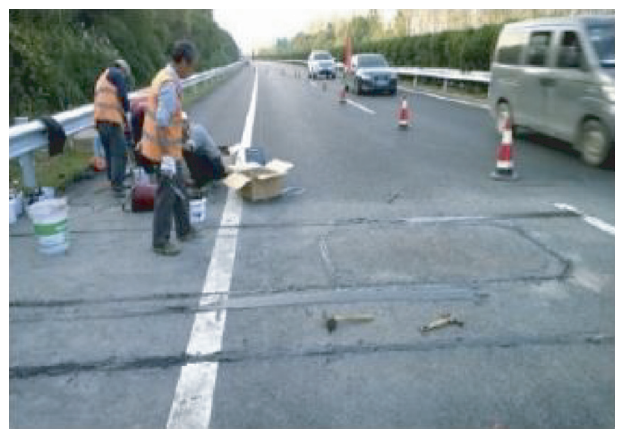

(a)

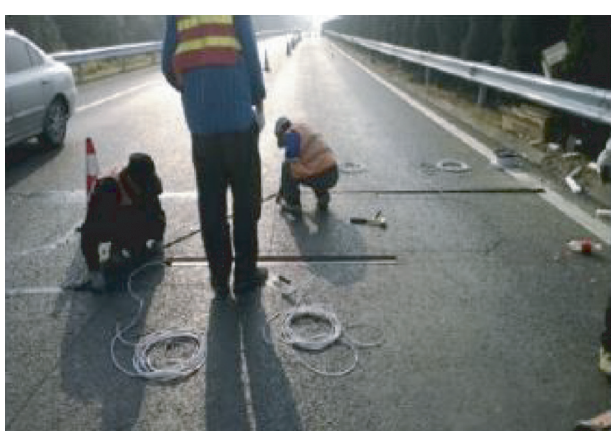

(b)

Figure 3: Installation of the field sensor for the vehicle pretest system.

TABLE 3: Calibration for speeds of different types of vehicles in the baseline state.

\begin{tabular}{lccccc}
\hline Vehicle type & Minibus & Coach bus & Light-duty truck & Medium-duty truck & Heavy-duty truck \\
\hline Vehicle speed $(\mathrm{km} / \mathrm{h})$ & 100 & 90 & 90 & 80 & 70 \\
\hline
\end{tabular}

TABle 4: Partial sample data: fuel consumption model.

\begin{tabular}{|c|c|c|c|c|c|c|}
\hline \multirow{2}{*}{ Serial number } & \multirow{2}{*}{ Roughness (m/km) } & \multicolumn{5}{|c|}{ Fuel consumption $(\mathrm{L} / 100 \mathrm{~km})$} \\
\hline & & Minibus & Coach bus & Light-duty truck & Medium-duty truck & Heavy-duty truck \\
\hline 1 & 1.25 & 7.23 & 25.32 & 18.32 & 24.68 & 32.87 \\
\hline 2 & 2.04 & 7.29 & 25.78 & 18.51 & 24.91 & 32.64 \\
\hline 3 & 2.31 & 7.42 & 25.8 & 18.7 & 25 & 32.8 \\
\hline 4 & 3.03 & 7.52 & 26.3 & 18.81 & 25.28 & 33.23 \\
\hline 5 & 1.06 & 7.19 & 25.4 & 18.21 & 24.62 & 32.45 \\
\hline 6 & 1.34 & 7.31 & 25.43 & 18.29 & 24.77 & 32.53 \\
\hline 7 & 1.54 & 7.18 & 25.55 & 18.38 & 24.72 & 32.67 \\
\hline 8 & 1.82 & 7.32 & 25.72 & 18.46 & 24.79 & 32.74 \\
\hline 9 & 1.13 & 7.2 & 25.42 & 18.35 & 24.62 & 32.52 \\
\hline 10 & 1.45 & 7.24 & 25.54 & 18.33 & 24.82 & 32.63 \\
\hline 11 & 3.24 & 7.65 & 26.31 & 18.76 & 25.24 & 33.22 \\
\hline 12 & 2.54 & 7.45 & 26.03 & 18.62 & 25.03 & 33.09 \\
\hline 13 & 1.35 & 7.23 & 25.52 & 18.18 & 24.73 & 32.58 \\
\hline 14 & 1.05 & 7.1 & 25.28 & 18.22 & 24.57 & 32.31 \\
\hline 15 & 0.94 & 7.16 & 25.34 & 18.16 & 24.49 & 32.48 \\
\hline 16 & 1.76 & 7.34 & 25.67 & 18.32 & 24.81 & 32.65 \\
\hline 17 & 1.64 & 7.35 & 25.97 & 18.19 & 24.79 & 32.72 \\
\hline 18 & 0.87 & 7.18 & 25.3 & 18.15 & 24.51 & 32.44 \\
\hline 19 & 1.23 & 7.22 & 25.54 & 18.27 & 24.58 & 32.49 \\
\hline 20 & 2.23 & 7.42 & 26 & 18.4 & 25.04 & 32.72 \\
\hline 21 & 2.67 & 7.49 & 26.04 & 18.73 & 25.24 & 33.14 \\
\hline 22 & 1.08 & 7.18 & 25.4 & 18.2 & 24.62 & 32.54 \\
\hline
\end{tabular}

correlation when they were fitted by applying an exponential function; however, to compare similar models and considering various factors including vehicle type and engine displacement, the model was still fitted by employing the linear function. The fuel consumption model is shown in Table 5.

According to the relationship between the fuel consumption of vehicles and the roughness at the baseline state in Table 5, the roughness was well correlated with the fuel consumption under the limits of the other constraints. By utilising the expression of the linear function, the fuel consumption increased with increasing IRI.

\section{Modified Fuel Consumption Model}

4.1. Modified Method. The relationship between roughness and fuel consumption in the baseline state was established. Considering that the conditions of the road surface varied spatially, the model in the baseline state was modified based on parameters (i.e., deflection and friction coefficient) of the road surface to enable the model to be suitable for different working conditions on the road surface.

It can be seen from the model at the baseline state that the relationships between fuel consumptions of five types of vehicles and the roughness were linear (as shown below). 
TABle 5: Roughness-fuel consumption model at the baseline state.

\begin{tabular}{lccc}
\hline Serial number & Vehicle type & Function expression & Correlation coefficient \\
\hline 1 & Minibus & $Q=0.191 \times$ IRI +6.976 & 0.908 \\
2 & Coach bus & $Q=0.440 \times$ IRI +24.91 & 0.910 \\
3 & Light-duty truck & $Q=0.281 \times$ IRI +17.90 & 0.866 \\
4 & Medium-duty truck & $Q=0.332 \times$ IRI +24.24 & 0.949 \\
5 & Heavy-duty truck & $Q=0.340 \times$ IRI +32.12 & 0.826 \\
\hline
\end{tabular}

Among models, the key parameters were $A$ and $B$; that is, the modification for the fuel consumption model was equivalent to modifying parameters $A$ and $B$.

$$
\mathrm{Q}=A \times \mathrm{IRI}+B .
$$

According to the independence tests carried out, the model was modified by applying different deflections and friction coefficients, that is, the influences of the deflection and friction coefficient on parameters $A$ and $B$. By taking the deflection as an example, the modified model can be expressed as follows:

$$
\begin{aligned}
A^{\prime} & =f(L, A) \\
\text { or } B^{\prime} & =f(L, B) .
\end{aligned}
$$

Parameters $A$ and $B$ are constants, so these functions can be expressed as the product of the original parameters $A$ and $B$ to simplify the model and calculation process as follows:

$$
\begin{aligned}
A^{\prime} & =f_{1}(L) \times A, \\
\text { or } B^{\prime} & =f_{2}(L) \times B .
\end{aligned}
$$

The influence functions of model modification based on deflection $f(L)$ on parameters $A$ and $B$ can be denoted as $k_{11}$ and $k_{12}$ and those of model modification based on the friction coefficient $f(S)$ on parameters $A$ and $B$ as $k_{21}$ and $k_{22}$, respectively. Therefore, the comprehensive influences of the above factors on parameters $A$ and $B$ can be expressed as $K_{1}$ and $K_{2}$, which are separately calculated as follows:

$$
\begin{aligned}
A^{\prime} & =f(L, S) \times A=K_{1} \times A=k_{11} \times k_{21} \times A, \\
B^{\prime} & =f(L, S) \times B=K_{2} \times B=k_{12} \times k_{22} \times B .
\end{aligned}
$$

According to the above expressions, the modification of the model in the baseline state was required to explore the influences of the deflection and friction coefficient on constants $A$ and $B$.

4.2. Model Modification Based on the Deflection and Friction Coefficients. Parameters $A$ and $B$ were calculated aiming at different types of vehicles based on the model in the baseline state and compared with those of the model at the baseline state. Moreover, the correlations of $k_{11}$ and $k_{12}$ with the defection $L$ as well as $k_{21}$ and $k_{22}$ with friction coefficient SFC were established. The calculated results are listed in Tables 6 and 7.

4.3. Modification of the Fuel Consumption Model Based on speed. The above model is established based on the calibrated speeds. Considering that reconstructed roads are restricted by various conditions such as traffic flow, the number of lanes, and speed limit, different types of vehicles are driven at different speeds. To improve the adaptability of the model to different working conditions, the model was further modified by focusing on vehicle speed.

The model was modified by only considering the speed. Owing to the related research models all being established in the baseline state, their speeds were defined correspondingly according to different vehicle types. The analysis was performed by focusing on the correction coefficient in the modification process. For convenience of calculation, the correction coefficient $m$ of the fuel consumption and the speed ratio $\lambda$ were taken as calculation indices. Based on the measured data, the correlation between the speed and fuel consumption of vehicles was analysed. By taking the minibus as an example, the relationship between the fuel consumption and the speed and that between $m$ and $\lambda$ are separately shown in Figures 4 and 5. Accordingly, a calculation model for the fuel consumption applicable to any working condition of reconstructed roads can be attained (Table 8).

\section{Calculation of Energy-Saving Benefit}

5.1. Calculation Method and Its Process. As mentioned above, based on the research object, i.e., reconstructed roads, the apparent parameters such as roughness of roads are improved due to reconstruction activity. This reconstruction activity is bound to bring corresponding energy-saving benefits for users who drive vehicles on these roads. Therefore, the differences of the fuel consumptions of users when they drive vehicles on roads before and after reconstruction were separately calculated based on the proposed calculation model to characterise the user benefit. The calculation formulae are as follows:

$$
\begin{aligned}
W & =\sum W_{k}, \\
W_{k} & =\left(\sum \Delta Q_{q i} \times \rho_{q} \times \delta_{q} \times S_{i}+\sum \Delta Q_{c j} \times \rho_{c} \times \delta_{c} \times S_{j}\right) \times 365,
\end{aligned}
$$

$\Delta Q_{q i}=Q_{q i}^{\prime \prime}-Q_{q i}^{\prime}$,

$\Delta Q_{c i}=Q_{c i}^{\prime \prime}-Q_{c i}^{\prime}$ 
TABLE 6: Correction parameters based on the deflection.

\begin{tabular}{lccccc}
\hline Vehicle type & Deflection & $A^{\prime}$ & $B^{\prime}$ & $k_{11}$ & $k_{12}$ \\
\hline \multirow{4}{*}{ Minibus } & 4.8 & 0.1909 & 6.7763 & 0.9994 & 0.9713 \\
& 5.3 & 0.1909 & 6.8723 & 0.9994 & 0.9848 \\
& 8.9 & 0.1910 & 6.9256 & 0.9995 & 0.9920 \\
& 15.5 & 0.1910 & 7.1372 & 1.0001 & 1.0221 \\
& 23.3 & 0.1911 & 7.0334 & 1.0001 & 1.0077 \\
\hline \multirow{4}{*}{ Coach bus } & 4.8 & 0.441 & 24.83 & 1.0023 & 0.9968 \\
& 5.3 & 0.436 & 24.89 & 0.9909 & 0.9992 \\
& 8.9 & 0.438 & 24.91 & 0.9955 & 1.0000 \\
Light-duty truck & 15.5 & 0.446 & 24.92 & 1.0136 & 1.0004 \\
& 23.3 & 0.451 & 25.01 & 1.0250 & 1.0040 \\
\hline & 4.8 & 0.271 & 16.23 & 0.9644 & 0.9067 \\
& 5.3 & 0.280 & 17.21 & 0.9964 & 0.9615 \\
& 8.9 & 0.278 & 17.92 & 0.9893 & 1.0011 \\
Medium-duty truck & 15.5 & 0.283 & 17.78 & 1.0071 & 0.9933 \\
& 23.3 & 0.285 & 18.02 & 1.0142 & 1.0056 \\
\hline & 4.8 & 0.321 & 23.21 & 0.9669 & 0.9575 \\
& 5.3 & 0.328 & 23.89 & 0.9880 & 0.9856 \\
& 15.9 & 0.330 & 24.21 & 0.9940 & 0.9988 \\
& 23.3 & 0.331 & 24.52 & 0.9970 & 1.0116 \\
& 0.353 & 25.70 & 1.0633 & 1.0602 \\
\hline & 4.8 & 0.328 & 30.46 & 0.9647 & 0.9483 \\
& 5.3 & 0.331 & 31.23 & 0.9735 & 0.9723 \\
& 8.9 & 0.341 & 32.32 & 1.0029 & 1.0062 \\
& 15.5 & 0.352 & 34.21 & 1.0353 & 1.0651 \\
& 23.3 & 0.363 & 35.23 & 1.0676 & 1.0968 \\
\hline
\end{tabular}

TABLE 7: Correction parameters based on the friction coefficient SFC.

\begin{tabular}{lccccc}
\hline Vehicle type & SFC & $A^{\prime}$ & $B^{\prime}$ & $K_{21}$ & $K_{22}$ \\
\hline \multirow{4}{*}{ Minibus } & 42 & 0.189 & 6.578 & 0.9895 & 0.9429 \\
& 48 & 0.189 & 6.821 & 0.9895 & 0.9778 \\
& 55 & 0.192 & 6.971 & 1.0052 & 0.9991 \\
& 58 & 0.193 & 7.182 & 1.0105 & 1.0292 \\
& 67 & 0.204 & 7.336 & 1.0681 & 1.0507 \\
\hline Coach bus & 42 & 0.434 & 24.20 & 0.9864 & 0.9715 \\
& 48 & 0.443 & 24.90 & 1.0068 & 0.9996 \\
& 55 & 0.452 & 25.12 & 1.0273 & 1.0084 \\
& 58 & 0.461 & 25.94 & 1.0477 & 1.0413 \\
Light-duty truck & 67 & 0.461 & 26.12 & 1.0477 & 1.0486 \\
\hline & 42 & 0.254 & 15.82 & 0.9039 & 0.8838 \\
& 48 & 0.281 & 17.65 & 1.0000 & 0.9860 \\
& 55 & 0.291 & 17.65 & 1.0356 & 0.9860 \\
& 58 & 0.302 & 18.12 & 1.0747 & 1.0123 \\
Medium-duty truck & 67 & 0.309 & 18.00 & 1.0996 & 1.0056 \\
\hline & 42 & 0.281 & 21.05 & 0.8464 & 0.8684 \\
& 48 & 0.329 & 24.43 & 0.9910 & 1.0078 \\
& 55 & 0.339 & 25.01 & 1.0211 & 1.0318 \\
& 58 & 0.381 & 25.92 & 1.1476 & 1.0693 \\
& 67 & 0.379 & 27.40 & 1.1416 & 1.1304 \\
\hline & 42 & 0.281 & 28.46 & 0.8265 & 0.8861 \\
& 48 & 0.345 & 28.27 & 1.0147 & 0.8801 \\
& 55 & 0.389 & 34.14 & 1.1441 & 1.0629 \\
& 58 & 0.412 & 36.45 & 1.2118 & 1.1348 \\
& 67 & 0.419 & 41.25 & 1.2324 & 1.2842 \\
\hline
\end{tabular}

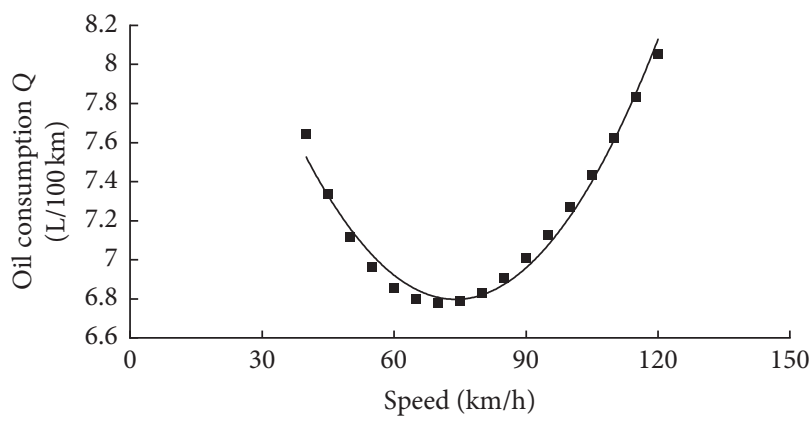

Figure 4: Fuel consumption vs. speed for the minibus.

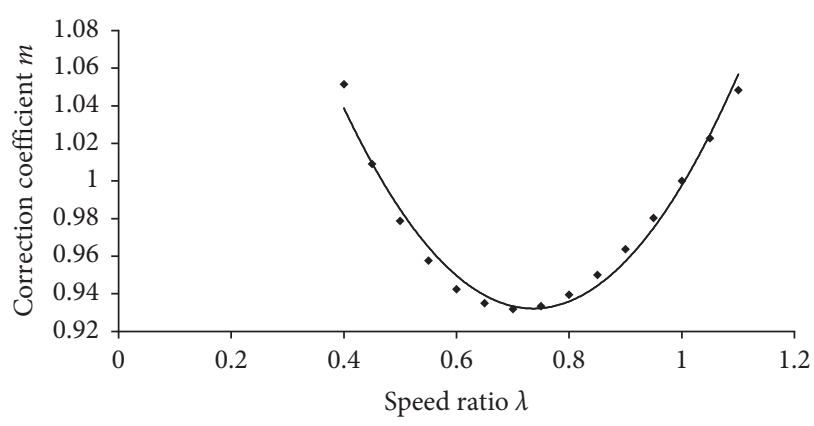

Figure 5: Correlation between $m$ and $\lambda$ for a minibus.

where $W$ and $W_{k}$ separately refer to users' energy-saving benefit (TCE) and the annual energy-saving benefit (TCE) within unit section $k ; \Delta Q_{q i}$ and $\Delta Q_{c i}$ denote the fuel-saving benefits $(L)$ of a single gasoline vehicle and a single diesel vehicle; $\rho_{q}$ and $\rho_{c}$ denote the densities of gasoline and diesel; $\delta_{q}$ and $\delta_{c}$ represent the standard coal equivalents (SCE) of gasoline and diesel; $S_{i}$ and $S_{j}$ refer to the driving distances of gasoline and diesel vehicles, respectively; $Q_{q i}^{\prime \prime}$ and $Q_{c i}^{\prime \prime}$ denote the fuel consumptions of a single gasoline vehicle and a single diesel vehicle after reconstruction; and $Q_{q i}^{\prime}$ and $Q_{c i}^{\prime}$ represent the fuel consumptions of a single gasoline vehicle and a single diesel vehicle before road reconstruction.

5.2. Case Application. According to the research, the model was applied to the Nanjing-Xuancheng highway reconstruction project, a green, circular, and low-carbon road project supported by special funds for energy saving and emission reduction in transportation. The related data before and after reconstruction were analysed, and the amount of annual energy saving of users was calculated (Table 9).

The user benefits in fuel saving and energy saving brought about by the implementation of reconstruction activity were reasonably evaluated by utilising the proposed model for calculating the user benefit in fuel consumption and the calculation method for the energy-saving benefit. The results indicated that improving the roughness of the road surface is important for improving driving comfort and users' travel benefit. 
TABLE 8: Modified model for roughness and fuel consumption.

\begin{tabular}{lcc}
\hline $\begin{array}{l}\text { Serial } \\
\text { number }\end{array}$ & Vehicle type & Function expressions \\
\hline 1 & Minibus & $Q=m \times\left(0.164 e^{0.003 S F C} \times\right.$ IRI $\left.+5.075 e^{0.002 L+0.004 S F C}\right), m=0.865 \lambda^{2}-1.28 \lambda+1.408$ and $\lambda=V / 100$ \\
2 & Coach bus & $Q=m \times\left(0.386 e^{0.001 L+0.002 S F C} \times\right.$ IRI $\left.+21.27 e^{0.003 S F C}\right), m=1.277 \lambda^{2}-1.984 \lambda+1.738$ and $\lambda=V / 90$ \\
3 & Light-duty truck & $Q=m \times\left(0.048 L^{0.005} S_{F C}^{0.413} \times\right.$ IRI $\left.+5.925 L^{0.023} S F C^{0.256}\right), m=1.492 \lambda^{2}-2.260 \lambda+1.805$ and $\lambda=V / 90$ \\
4 & Medium-duty truck & $Q=m \times\left(0.170 e^{0.004 L+0.011 S F C} \times\right.$ IRI $\left.+13.886 e^{0.004 L+0.009 S F C}\right), m=2.933 \lambda^{2}-4.142 \lambda+2.382$ and $\lambda=V / 80$ \\
5 & Heavy-duty truck & $Q=m \times\left(0.147 e^{0.007 L+0.015 S C} \times\right.$ IRI $\left.+12.739 e^{0.005 L+0.016 S F C}\right), m=3.071 \lambda^{2}-4.133 \lambda+2.348$ and $\lambda=V / 70$ \\
\hline
\end{tabular}

TABle 9: Calculation of users' energy-saving benefits from the Nanjing-Xuancheng highway reconstruction work.

\begin{tabular}{lcc}
\hline Type of fuels & Gasoline & Diesel \\
\hline Cumulative annual fuel saving (L) & 269,757 & 703,320 \\
Density (kg/L) & 0.725 & 0.835 \\
Cumulative annual fuel saving (t) & 195.57 & 587.27 \\
SCE & 1.4714 & 1.4571 \\
Cumulative annual energy saving (TCE) & 287.77 & 855.71 \\
Annual energy saving (TCE) & \multicolumn{2}{c}{1143.48} \\
\hline
\end{tabular}

\section{Conclusion}

The calculation method for the fuel consumption model of users' vehicles was proposed based on the roughness of the reconstructed roads. The following conclusions are drawn:

(1) The couple of rolling resistance of vehicles triggered by the wavelength of macrostructures for characterising the roughness of road surfaces was a main factor influencing the fuel consumption of vehicles. The larger the IRI, the greater the fuel consumption of vehicles.

(2) The test and acquisition schemes for the fuel consumption of vehicles and parameters of the road surfaces were formulated. Moreover, according to the measured data, a single-parameter model for the fuel consumption of vehicles and the roughness at the baseline state was established.

(3) Aiming at the deflection, friction coefficient, and vehicle speed, a modified model for the roughness and fuel consumption of vehicles was established; in addition, the calculation method for the users' energy-saving benefit from reconstructed roads and its process were proposed: a case study was also analysed, which exerts reference and guidance significance for evaluating the users' energy-saving benefit from reconstructed roads.

\section{Data Availability}

All the data used to support the findings of this study are available from the corresponding author upon request.

\section{Conflicts of Interest}

The authors declare that they have no conflicts of interest.

\section{References}

[1] Ministry of Transport of the People's Republic of China, Annual Report on Green Cycle Low Carbon Transportation Development, People's Communications Press, Beijing, China, 2013.

[2] Department of Transportation of Jiangsu Province (China), Development Plan of Green Cycle Low Carbon Transportation in Jiangsu Province (2013-2020), Department of Transportation of Jiangsu Province, Nanjing, China, 2013.

[3] D. P. Yang, "Study on evaluation of vehicle fuel consumption and emission in different highway grades," Science Technology and Engineering, vol. 17, pp. 108-114, 2017.

[4] N. Lan, "Study on fuel consumption characteristics of truck at constant speed," Highway and Automobile Transportation, vol. 1, pp. 9-12, 2018.

[5] Z. S. Yu, Automobile Theory, China Machinery Press, Beijing, China, 1990.

[6] C. Bester, "The effect of pavement type and condition on the fuel consumption of vehicles," Transportation Research Record, vol. 1000, pp. 28-32, 1984.

[7] P. Cenek, N. Jamieson, and G. Ball, "Effect of pavement deflection on rolling resistance of commercial vehicle tyres," in Proceedings of the 3rd International Symposium on Pavement Surface Characteristics, ARRB Transport Research Ltd, Christchurch, New Zealand, September 1996.

[8] F. Ross, "Effect of pavement roughness on vehicle fuel consumption," Transportation Research Record, vol. 846, pp. 1-6, 1982.

[9] P. Sumitsawan, S. Ardekani, and S. Romanoschi, "Effect of pavement type on fuel consumption and emissions," in Proceedings of the Mid-Continent Transportation Research Symposium, 2009, Iowa State University, Ames, IA, USA, August 2009.

[10] L. F. Hong, "Experimental study on influencing factors of fuel consumption in automobile transportation," Journal of Highway and Transportation Research and Development, vol. 8, 1991.

[11] Research Report ARR 314.ARRB Transport Research Ltd.

[12] Ministry of Transport of the People's Republic of China, Code for Design of Highway Asphalt Pavement, Ministry of Transport of the People's Republic of China, Beijing China, 2006.

[13] Ministry of Transport of the People's Republic of China, Evaluation Standard of Highway Technical Condition, Ministry of Transport of the People's Republic of China, Beijing, China, 2007.

[14] H. F. Jia, "Determination and comparison of fuel consumption index in expressway post evaluation," Journal of Jilin University, vol. 34, 2004. 